\title{
Searches for possible supernovae - GRBs correlations
}

\author{
R. Hudec ${ }^{1}$, V. Hudcová ${ }^{1}$, and F. Hroch ${ }^{2}$ \\ 1 Astronomical Institute, CZ-251 65 Ondřejov, Czech Republic \\ 2 Dept. of Theoretical Physics and Astrophysics, Masaryk University Brno, Czech Republic
}

Received January 21; accepted May 10, 1999

\begin{abstract}
We present the first preliminary results of a cross correlation between the 4B GRB catalog and the catalog of supernovae detected in the same time period. No restrictions and/or limitations have been applied. The goal is to select SNe which may, in fact, represent unrecognized optical afterglows of GRBs.
\end{abstract}

Key words: gamma-ray: bursts

\section{Introduction}

The recent detections of optical afterglows of Gamma Ray Bursts (GRBs) confirm the low energy emission of these events. There has been optical afterglows observed for 7 GRBs so far, hence limited information is available now for their light curves, magnitudes as well as other parameters.

The common properties of optical afterglows (further OA) can be summarized as follows.

1. The OA peak at roughly mag $14-23$.

2. They exhibit power law declines with mean power of -1.2 .

3. They seem to be related to faint host galaxies (but not in all cases).

4. Not all of the GRBs have an associated OA.

5. Spectra of OAs remain poorly investigated.

These general properties and behavior of OA resemble to some extent those of supernovae especially by peak magnitudes, transient behavior, and also by mean decline rates. Further, the rate of OA may be much larger than the GRBs rate due to different beaming, this factor is unknown but may reach 10 or even 100. It is therefore obvious that some of the recently detected and poorly in investigated supernovae may, in fact, represent unrevealed OA.

Send offprint requests to: $\mathrm{R}$. Hudec
There are however also indications that there may be even direct physical relations between (some) GRBs and (some) SN, as illustrated by the positional and temporal coincidence of SN 1998bw with GRB 980425. This is why we have decided to study the possible correlations of GRBs and SN in more detail. In this search, we have taken all SNe detected into account including faint and poorly investigated events as they may represent unrevealed OA. Our approach is to study in detail the coincidences found and SN in question rather than make pure statistical conclusions (which are difficult due to large positional inaccuracies of GRBs and other influences such as incompleteness of SN databases). We note that the incompleteness of the SNe catalogues is large and that only $10^{-3} \ldots 10^{-4}$ of all SNe down to mag 23 are included.

\section{Data}

The 4B GRB catalog available at the www (Paciesas et al. 1998) has been used as the source of GRBs parameters and positions. This database has been cross-correlated with the list of supernovae compiled by us over the whole time interval of the $4 \mathrm{~B}$ catalog from reports published in the IAUC. Since the OA are known to peak roughly one day after the corresponding GRB and then declining gradually, and since the first SN observations are usually delayed after their maxima, we have searched for correlations between GRBs and supernovae detected within the particular GRB error boxes up to 30 days after the gamma ray events (for a more complex approach including searches for SNe detected before GRB triggers see Hudec et al. 1999).

Altogether 2133 GRBs (BATSE triggers 105 to 6720 ) and 613 SN detected between April 21, 1991 and May 3, 1998 have been analyzed.

\section{Preliminary results}

The number of correlations found (24 SN and 29 GRBs in five cases there are two GRBs consistent with particular $\mathrm{SNe}$ ) is consistent with the assumption of unrelated samples (both statistical as well as systematic errors of GRBs 


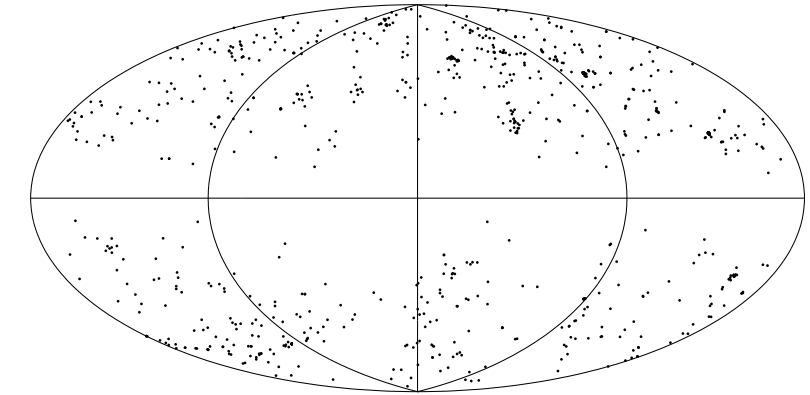

Fig. 1. Angular distribution of historical supernovae in galactic coordinates

taken into account; for more details on the statistical procedures see Hudec et al. 1999). Moreover, we have considered the possible correlations with GRBs of SN 1990aj and SN 1993R (Patat \& Piemonte 1998), based on their spectra similar to SN 1998bw. The SN 1993R is included in the list of positive correlations and may be related to GRB 930524; although this correlation is rather weak due to the large error box of this GRB. For the SN 1990aj, no positional GRB catalogues are available.

\subsection{Examples of correlations found}

The full results and their discussion will be published elsewhere (Hudec et al. 1999). In the following we list a few most promising correlations.

\subsubsection{SN 1998B}

The supernova reached $\sim 18.5$ mag at the time of detection (Jan. 1, 1998). Spectrum SNIa 3-4 weeks after maximum, $z=0.045$, possibly related to GRB 971218 .

\subsubsection{SN 1996bx}

$R \sim 19.2$ at the time of detection (Nov. 18, 1996), SN Ia 3 -4 weeks after maximum, $z=0.058$, possibly related to GRB 961029.

\subsubsection{SN 1995bc}

$\sim 18.5$ mag at the time of the detection (Dec. 1, 1995), spectrum SN II, $z=0.048$, possibly related to GRB 951107.

\subsubsection{SN 1991aa}

$\sim 16$ mag at the time of the detection (May 7, 1991), spectrum SN Ib, possibly related to GRB 910423.

\subsubsection{SN 1992Q}

$\sim 18.5 \mathrm{mag}$ at the time of the detection (April 7, 1992), blue object, possibly related to GRB 920321.

\section{Discussion}

Our search is more general than those of Kippen et al. (1998) and Wang \& Wheeler (1998): we consider all known SNe without any restriction since any limitation would be in contrast with the main goal of our study (search for possible unrecognized OAs of GRBs in the sample of detected $\mathrm{SNe}$ ). It should be also noted that the observed SNe, like GRBs, shows an isotropic sky distribution (Fig. 1). The study of Kippen et al. (1998) is restricted to brighter events only (brighter than mag 17) but this is in contrast with BeppoSAX OA statistics (their peaks are below $17 \mathrm{mag}$ in all detected 7 cases) while Wang and Wheeler (1998) restrict to SNe of known Ia, Ib types (but the OA may be also among weak and unclassified SNe).

The statistical significance of the results of crosscorrelations between the GRBs and SNe is affected and limited by the following: (1) the positional uncertainty of a quite large fraction of the GRBs is rather high (2) the dates of SN peaks are unknown in most cases (3) the results of optical SNe searches do not represent a full and homogeneous sample so that many (and even a large majority) of the SNe may be missed especially at faint magnitudes (4) there is no systematic sky patrol survey at magnitudes below 15 .

For the correlations found, most of the related SNe represent poorly investigated events with poorly known (and in many cases even completely unknown) light curves, decline rates, color indices and no or limited spectral information hence the decision whether they could be related to the GRBs in question is difficult. The recent detections of faint OA of GRBs strongly support and justify further more extended and more complete searches for faint new and variable optical objects especially at low magnitudes (18 and less).

Some of this information can be retrieved from deep archival plates (some of the archival plate collections reach the limiting magnitudes of 20 and even 23 such as ROE Edinburgh and TLS Tautenburg) but a systematic deep CCD patrol could provide more precise and much more complete database.

Acknowledgements. The work has been supported by the Projects of the Czech Ministry for Education and Youths, Nos. ES02 and ES036.

\section{References}

Hudec R., et al., 1999, A\&A (in preparation)

Paciesas W.S., et al., 1998, astro-ph/9903205, http://www.batse.msfc.nasa.gov/batse/grb/catalog

Patat F., Piemonte A., 1998, IAUC 7071

Kippen R.M., et al., 1998, ApJ 506, L27

Wang L., Wheeler J.C., 1998, ApJ 504, L87 